\title{
Constructing multilayers with absorbing materials
}

\author{
Juan I. Larruquert*, Manuela Vidal-Dasilva, Sergio García-Cortés, \\ Mónica Fernández-Perea, José A. Méndez, and José A. Aznárez \\ GOLD-Instituto de Física Aplicada, CSIC, Serrano 144, 28006 Madrid, Spain \\ *E-mail: larruquert@ifa.cetef.csic.es \\ Received October 31, 2009
}

\begin{abstract}
The strong absorption of materials in the extreme ultraviolet (EUV) above $\sim 50 \mathrm{~nm}$ has precluded the development of efficient coatings. The development of novel coatings with improved EUV performance is presented. An extensive research was performed on the search and characterization of materials with moderate absorption, such as various lanthanides. Based on this research, novel multilayers based on $\mathrm{Yb}$, $\mathrm{Al}$, and $\mathrm{SiO}$ have been developed with a narrowband performance in the 50-92 nm range. Furthermore, procedures for the design of multi-material multilayers with absorbing materials have been derived, which resulted in multilayers with enhanced reflectance.
\end{abstract}

OCIS codes: 230.4170 , 260.7200, 340.7470, 350.6090, 220.3740.

doi: 10.3788/COL201008S1.0159.

Grupo de Optica de Láminas Delgadas (GOLD) is a laboratory devoted to the research of coatings for the far ultraviolet (FUV) (105-200 nm) and extreme (EUV) $(12-105 \mathrm{~nm})$ GOLD is capable of preparing the standard coatings for FUV and EUV. Furthermore, GOLD also works towards developing novel coatings with challenging throughput for this same range.

EUV and, to some extent, FUV have been lacking efficient coatings, with both broadband and narrowband performance, because the overwhelming part of materials is burdened with a strong absorption above $\sim 50 \mathrm{~nm}$. Hence, single-layer coatings (sometimes coated with a protective overlayer) are often used.

The standard coatings that can be prepared at GOLD include: broadband reflective coatings for the EUV, such as sputter-deposited $\mathrm{SiC}$; broadband reflective coatings for the FUV, such as Al protected with $\mathrm{MgF}_{2}$. narrowband transmittance filters for the FUV based on multilayers of $\mathrm{Al}$ and $\mathrm{MgF}_{2}$.

In addition to these, GOLD has been investigated on novel coatings for the EUV-FUV, including narrowband multilayers tuned in the difficult 50-92-nm range based on $\mathrm{Al}$ and $\mathrm{Yb}, \mathrm{Al} / \mathrm{MgF} 2 / \mathrm{SiC}$ broadband reflective coatings that retain the high EUV reflectance of $\mathrm{SiC}$ with an enhanced reflectance above $105 \mathrm{~nm}$.

An important part of GOLD research has been devoted to the characterization of materials in the EUV-FUV, i.e., to measuring the optical constants $n$ (refractive index) and $k$ (extinction coefficient) of materials in this range. This research has provided GOLD with an extensive knowledge on the optical properties of materials in this range, which has given rise to the development of some novel coatings

The deposition techniques available at GOLD are evaporation (filaments and electron beam) and ion beam sputtering (IBS). These techniques can be combined to deposit multilayer alternating films in deposition chambers that work under ultrahigh vacuum, which enable the coating deposition of reactive materials. The system combining evaporation and ion beam sputtering is connected via a vacuum to a reflectometer covering the range of 12-200 $\mathrm{nm}$. The reflectometer-deposition sys- tem has been described ${ }^{[1,2]}$.

Here, research on multilayer coatings for the EUVFUV is reported. Some examples of the performance of standard coatings are presented firstly. Then, we present novel narrowband multilayers developed for the long EUV. Finally, enhanced-reflectance multilayers based on the superposition of absorbing materials are described.

The reflective coatings of $\mathrm{Al}$ protected with $\mathrm{MgF}_{2}$, both materials deposited by thermal evaporation, have been the best established coating of FUV for over 40 years ${ }^{[3]}$. They can be prepared with a reflectance above $80 \%$ at wavelengths longer than $\sim 120 \mathrm{~nm}$. Preparing best-performance $\mathrm{Al} / \mathrm{MgF}_{2}$ coatings, however, is not straightforward because $\mathrm{Al}$ is easily oxidized even under high vacuum so that it has to be protected readily, and $\mathrm{MgF}_{2}$ must be free of impurities in order to keep its high transmittance down to $115 \mathrm{~nm}$, the $\mathrm{MgF}_{2}$ cutoff. Figure 1 displays the reflectance of an $\mathrm{Al} / \mathrm{MgF}_{2}$ coating once exposed to the atmosphere.

Ion beam sputter-deposited $\mathrm{SiC}$ (IBS-SiC) is the preferred coating when a high reflectance is required in the $\sim 70-120 \mathrm{~nm}$ range of FUV-EUV. IBS-SiC often replaces the somewhat more efficient chemical vapordeposited $\mathrm{SiC}$ (CVD-SiC) because the former involves a low-temperature process and hence a lower cost; the high temperature required for CVD-SiC is not suitable for coating conventional mirrors and diffraction gratings. Furthermore, CVD-SiC grows as a rough coating, the polishing of which is complicated due to the hardness of SiC. Figure 2(a) displays the in situ reflectance of a freshly deposited, 38-nm-thick $\mathrm{SiC}$ film prepared at $\mathrm{GOLD}^{[4]}$ and measured at six angles away from the normal. Data are an average of measurements performed in two perpendicular planes of incidence, so that the average reflectance over the two planes represents the reflectance that would be measured for non-polarized incoming radiation. Figure $2(\mathrm{~b})^{[4]}$ displays the near-normal reflectance of a 64-nm-thick $\mathrm{SiC}$ film. Data correspond to measurements performed in situ, after a short exposure to the atmosphere and after prolonged storage in a desiccator. Reflectance displays a noticeable drop when shortly exposed to the atmosphere, whereas this drop 


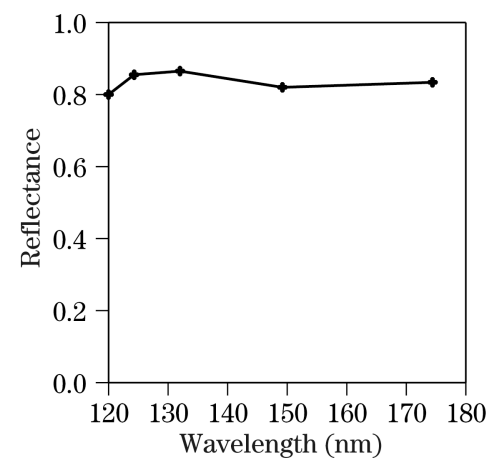

Fig. 1. Reflectance of an $\mathrm{Al} / \mathrm{MgF}_{2}$ coating optimized for the largest reflectance at $\sim 122 \mathrm{~nm}$.
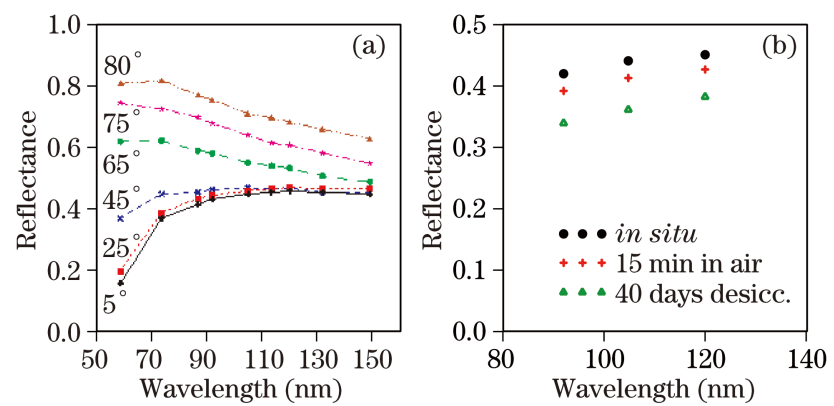

Fig. 2. (color online) (a) Reflectance versus wavelength of a fresh IBS-SiC film for various angles of incidence measured away from the normal to the sample; (b) In situ reflectance versus the wavelength of a fresh IBS-SiC film and decay over time.

sharply slows down over time; the reflectance after a period of 40 days in a desiccator is expected to approach its lowest values, with no significant further decay. This limited reflectance decrease is due to the slight oxidation of IBS-SiC films ${ }^{[5]}$.

Our search for materials with high reflectance found single boron (B) films as an interesting coating for EUV $^{[6]}$. We prepared and characterized e-beam evaporated $\mathrm{B}$ films with near-normal reflectance similar to those of IBS-SiC and IBS-B ${ }_{4}$ C. A preliminary aging test of $\mathrm{B}$ films showed a reflectance degradation ranging from moderate to large depending on the sample; more research is required to better establish the aging of $B$ films.

Coatings with narrowband reflection properties have become available in most spectral ranges. However, the 50-115 nm spectral range of EUV has been lacking such coatings because the overwhelming part of materials is burdened with a strong absorption; this makes the accumulation of layers in a multilayer inefficient because radiation is mostly absorbed in the outermost layer.

A source of relatively low absorption materials in this spectral range has been found among lanthanides, and many of them have been fully characterized recently $\left(\mathrm{La}^{[7]}, \mathrm{Tb}^{[8]}, \mathrm{Gd}^{[9]}, \mathrm{Nd}^{[9]}, \mathrm{Yb}^{[10,11]}, \mathrm{Ce}^{[12]}, \mathrm{Pr}^{[13]}, \mathrm{Eu}^{[14]}\right.$, $\mathrm{Tm}^{[15]}$, and $\mathrm{Dy}^{[16]}$, along with materials with close chemical properties such as $\mathrm{Sc}^{[17-20]}$. This relatively low absorption makes them candidates for use in novel multilayer coatings for this spectral range.

The number of narrowband reflectance multilayers developed so far within the $\sim 50-115 \mathrm{~nm}$ spectral range

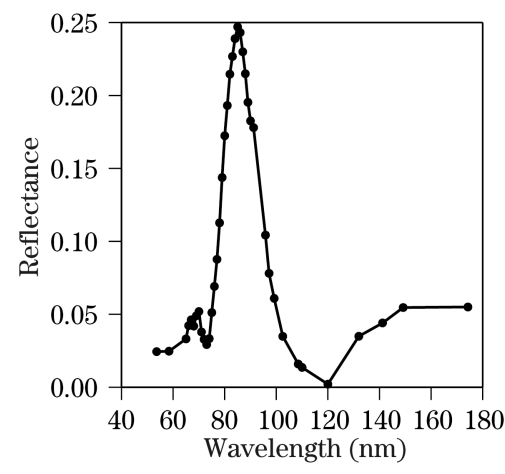

Fig. 3. Reflectance versus the wavelength of a fresh $\mathrm{Yb} / \mathrm{Al} / \mathrm{Yb} / \mathrm{SiO}$ multilayer.

is very limited. Narrowband multilayers based on $\mathrm{Tb}^{[8,21,22]}, \mathrm{Gd}^{[9]}, \mathrm{Nd}^{[9]}$, and $\mathrm{La}^{[22]}$, combined with $\mathrm{Si}$, $\mathrm{SiC}$, or $\mathrm{B}_{4} \mathrm{C}$, have been developed recently. These multilayers demonstrated useful bands centered in the $\sim 55$ $69 \mathrm{~nm}$ range with peak reflectances in the $0.12-0.27 \mathrm{~nm}$ range. At wavelengths longer than $\sim 69 \mathrm{~nm}$, a multilayer based on $\mathrm{La}$ and $\mathrm{B}_{4} \mathrm{C}$, with a peak reflectance of $\sim 0.118$ at $92 \mathrm{~nm}$, has been reported ${ }^{[22]}$.

Among lanthanides, $\mathrm{Yb}$ has the smallest absorption in the $\sim 60-105 \mathrm{~nm}$ range. For this reason, $\mathrm{Yb}$ was selected as one of the materials for GOLD's novel multilayers. We have developed a new type of multilayer coating tuned in the $\sim 50-92 \mathrm{~nm}$ range ${ }^{[23]}$, which is based on the relative transparency of $\mathrm{Yb}$ and the use of $\mathrm{Al}$ as a contrasting material; $\mathrm{SiO}$ is the third material of the multilayer, and it was used initially as a protective material and finally also for barrier layers between $\mathrm{Al}$ and $\mathrm{Yb}$.

Multilayers of $\mathrm{Yb} / \mathrm{Al} / \mathrm{Yb} / \mathrm{SiO}$, starting with the innermost layer, were found to provide valuable filtering performance. The different films of the multilayers were deposited by thermal evaporation in ultrahigh vacuum conditions. The reflectance band was found to shift toward shorter wavelengths when the angle moved away from normal incidence. Figure 3 displays the reflectance measured for a freshly deposited $\mathrm{Yb} / \mathrm{Al} / \mathrm{Yb} / \mathrm{SiO}$ multilayer.

On the negative side, a dendrite structure was detected under the microscope, which was attributed to the reactivity between $\mathrm{Al}$ and $\mathrm{Yb}$. A thin $\mathrm{SiO}$ barrier layer between $\mathrm{Al}$ and $\mathrm{Yb}$ was found effective to avoid the formation of the dendrite structure.

A theory was derived to design multilayer coatings with the maximum reflectance at a desired wavelength. The difference with standard multilayers is that the new multilayers must deal with films of highly radiationabsorbing materials, and a reflectance enhancement is obtained over the reflectance of single-layer coatings ${ }^{[24,25]}$. Although the obtained mathematical derivations are valid in any spectral range, they are particularly adequate for EUV, where transparent materials for standard multilayers are inexistent or marginally available. According to this theory, reflectance enhancements can be obtained by the superposition of thin films of various materials with certain material selection rules.

The main material selection rule can be easily interpreted through a graphical representation. Let $N=n$ $+j k$ be the complex refractive index of an absorbing material. Let us plot the refractive indices of all available 

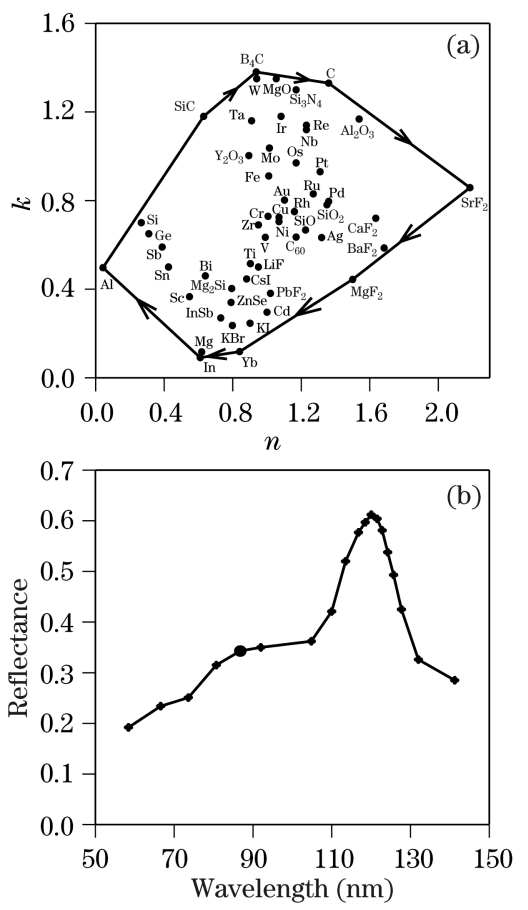

Fig. 4. (a) Representation in the complex plane of the refractive indices of many materials at $91.2 \mathrm{~nm}$; (b) reflectance of an $\mathrm{Al} / \mathrm{MgF}_{2} / \mathrm{SiC}$ multilayer aged three years.

materials at the desired wavelength in the $n-k$ complex plane. Figure 4(a) shows the refractive indices of many materials at $91.2 \mathrm{~nm}$. The material selection rule in order to obtain a reflectance enhancement with a multilayer is satisfied when we switch from one material to the next underneath in the multilayer by clockwise rotation in the $n-k$ plane, that is, by selecting the materials in the direction given by the arrows going inwards. A polygon is formed with the most extreme optical constants plotted in Fig. 4(a). A multilayer with $\mathrm{SiC}$ as the outer material and with the sequence $\mathrm{B}_{4} \mathrm{C}, \mathrm{C}, \mathrm{SrF}_{2}$, and $\mathrm{MgF}_{2}$, among others (going inwards), would provide us, in principle, with the largest possible reflectance at $91.2 \mathrm{~nm}$. Theoretically, the more materials with extreme optical constants deposited in the correct sequence, the larger the reflectance of the multilayer at the desired wavelength will be. In practice, the difficulty of depositing many different materials and the saturation of reflectance enhancement when many materials are involved (among other difficulties) suggest limiting the number of materials from $\sim 3$ to 4. Several combinations of materials $\left(\mathrm{Al} / \mathrm{MgF}_{2} / \mathrm{SiC}\right.$, $\mathrm{Al} / \mathrm{MgF}_{2} / \mathrm{B}_{4} \mathrm{C}$, and $\mathrm{C} / \mathrm{B}_{4} \mathrm{C} / \mathrm{SiC}$ ) have been attempted so far ${ }^{[26,27]}$.

We have been working on the development of this kind of multilayer, and have started with $\mathrm{Al} / \mathrm{MgF}_{2} / \mathrm{SiC}$ ( $\mathrm{SiC}$ is the outermost layer; only one layer per material); the reflectance of a three-year-old multilayer coating prepared at GOLD is plotted in Fig. 4(b). Although the multilayer was optimized for the largest reflectance at $91.2 \mathrm{~nm}$ (the filled circle in the figure highlights the measurement at the close wavelength of $92.0 \mathrm{~nm}$ ), the reflectance increase was much larger at $\sim 122 \mathrm{~nm}$ due to the transparency of $\mathrm{MgF}_{2}$ above $115 \mathrm{~nm}$ and the high reflectance of $\mathrm{Al}$. Hence, this multilayer provides a reflectance as high as or slightly higher than the single-layer $\mathrm{SiC}$ coatings be- low $\sim 105 \mathrm{~nm}$ and a large reflectance increase over $\mathrm{SiC}$ films above $105 \mathrm{~nm}$.

We can look at multi-material multilayers with absorbing materials as a kind of generalization of both (1) standard two-material multilayers and (2) single-layer coatings ${ }^{[24,25]}$. Let us refer to these multilayers as subquarter-wave multilayers (SQWMs) due to the fact that the films are generally thinner than quarter waves). Let us assume that in the multilayer, we use $m$ different materials with refractive indices $N_{1}$ to $N_{m}$. When going inwards in the multilayer and after making use of all $m$ refractive indices, we can start again with $N_{1}$, completing a full period; then we can continue adding more periods underneath as long as the reflectance enhancement is worth adding more layers. Hence, SQWMs are a generalization of standard two-material multilayers to any number of materials above two.

1) For standard two-material multilayers, one full period is completed after two layers, whereas in SQWM, it is completed after $m$ layers.

2) For standard two-material multilayers, the refractive index oscillates between two values, whereas in SQWM, it must rotate in the correct sequence among $N_{i}, i=1$ to $m$ indices.

3) The integrated optical path through one full period is $\lambda / 2$ for both standard multilayers (shared by the two layers in a bilayer) and SQWM (shared by all $m$ layers in a period; this explains the name of SQWM).

Furthermore, SQWMs are also a generalization of the case of a single-layer coating, which can be understood with the following example. Let us assume an SQWM with several materials and only one period, such as few multilayers developed for the $50-105 \mathrm{~nm}$ range ${ }^{[26],[27]}$; this multilayer can be simplified step by step by removing one layer (and material) at a time (and reducing reflectance correspondingly) until a single-layer coating is left.

In addition to efficient SQWM in the 50-115 nm range, designs tuned at the wavelengths of $50,30.4^{[28]}, 13.4$, and $11.3^{29,30]} \mathrm{nm}$ have been proposed, with reflectance enhancements over standard two-material multilayers.

The SQWM with the highest possible reflectance at a desired wavelength has a depth-graded thickness distribution. For s or p polarized radiation and for normal incidence with any polarization, the layer thicknesses of the multilayer with the largest reflectance at a given wavelength can be optimized sequentially from the innermost to the outermost layer, so that it is not necessary to perform a new optimization of the entire stack when new layers are added on top of an optimized system ${ }^{[30-33]}$.

This work was supported by the National Programme for Space Research, Subdirección General de Proyectos de Investigación, Ministerio de Ciencia e Innovación (Nos. ESP2005-02650 and AYA2008-06423-C03-02/ESP: AYA2009-14070). M. V. is thankful to Ministerio de Educación y Ciencia for the funding under the FPI BES-2006-14047 fellowship. S. García-Cortés and M. Fernández-Perea are thankful to Consejo Superior de Investigaciones Científicas (Spain) for funding under the Programa I3P, partially supported by the European Social Fund. At present, M. Fernández-Perea is at Lawrence Livermore National Laboratory, 7000 East 
Avenue, Livermore, California 94550, USA. We likewise acknowledge the technical assistance of José M. SánchezOrejuela.

\section{References}

1. J. A. Aznärez, J. I. Larruquert, and J. A. Mëndez, Rev. Sci. Instrum. 67, 497 (1996).

2. J. I. Larruquert, J. A. Aznädez, and J. A. Mëndez, Proc. SPIE 4139, 92 (2000).

3. G. Hass and R. Tousey, J. Opt. Soc. Am. 49, 593 (1959).

4. M. Fernändez-Perea, J. A. Mëndez, J. A. Aznärez, and J. I. Larruquert, Appl. Opt. 48, 4698 (2009).

5. D. Schwarz and R. A. M. Keski-Kuha, Mat. Res. Soc. Symp. Proc. 396, 503 (1996).

6. M. Vidal-Dasilva, M. Fernändez-Perea, J. A. Mëndez, J. A. Aznärez, and J. I. Larruquert, Appl. Opt. 47, 2926 (2008)

7. Y. Uspenski, J. Seely, N. Popov, I. Artioukov, A. Vinogradov, D. Windt, and B. Kjornrattanawanich, Proc. SPIE 5919, 213 (2005).

8. D. L. Windt, J. F. Seely, B. Kjornrattanawanich, and Y. A. Uspenskii, Opt. Lett. 30, 3186 (2005).

9. B. Kjornrattanawanich, D. L. Windt, Y. A. Uspenskii, and J. F. Seely, Proc SPIE 6317, 63170U (2006).

10. J. I. Larruquert, J. A. Aznärez, J. A. Mëndez, and J. Calvo-Angös, Appl. Opt. 42, 4566 (2003).

11. M. Fernändez-Perea, J. I. Larruquert, J. A. Aznärez, J. A. Mëndez, L. Poletto, D. Garoli, A. M. Malvezzi, A. Giglia, and S. Nannarone, J. Opt. Soc. Am. A 24, 3691 (2007).

12. M. Fernändez-Perea, J. I. Larruquert, J. A. Aznärez, J. A. Mëndez, L. Poletto, D. Garoli, A. M. Malvezzi, A. Giglia, and S. Nannarone, J. Appl. Phys. 103, 073501 (2008).

13. M. Fernändez-Perea, M. Vidal-Dasilva, , J. A. Aznärez, J. I. Larruquert, J. A. Mëndez, L. Poletto, D. Garoli, A. M. Malvezzi, A. Giglia, and S. Nannarone, J. Appl. Phys. 103, 113515 (2008).

14. M. Fernändez-Perea, M. Vidal-Dasilva, J. A. Aznärez, J. I. Larruquert, J. A. Mëndez, L. Poletto, D. Garoli, A. M.
Malvezzi, A. Giglia, and S. Nannarone, J. Appl. Phys. 104, 123527 (2008).

15. M. Vidal-Dasilva, M. Fernändez-Perea, J. A. Aznärez, J. I. Larruquert, J. A. Mëndez, L. Poletto, A. M. Malvezzi, A. Giglia, and S. Nannarone, J. Appl. Phys. 105, 103110 (2009).

16. B. Kjornrattanawanich, D. L. Windt, J. A. Bellotti, and J. F. Seely, Appl. Opt. 48, 3084 (2009).

17. A. L. Aquila, F. Salmassi, E. M. Gullikson, F. Eriksson, and J. Birch, Proc. SPIE 5538, 64 (2004).

18. Y. A. Uspenskii, J. F. Seely, N. L. Popov, A. V. Vinogradov, Y. P. Pershin, and V. V. Kondratenko, J. Opt. Soc. Am. A 21, 298 (2004).

19. J. I. Larruquert, J. A. Aznärez, J. A. Mëndez, A. M. Malvezzi, L. Poletto, and S. Covini, Appl. Opt. 43, 3271 (2004).

20. M. Fernändez-Perea, J. I. Larruquert, J. A. Aznärez, J. A. Mëndez, L. Poletto, A. M. Malvezzi, A. Giglia, and S. Nannarone, J. Opt. Soc. Am. A 23, 2880 (2006).

21. B. Kjornrattanawanich, D. L. Windt, J. F. Seely, and Y. A. Uspenskii, Appl. Opt. 45, 1765 (2006).

22. J. F. Seely, Yu. A. Uspenskii, B. Kjornrattanawanich, and D. L. Windt, Proc. SPIE 6317, 63170T (2006).

23. M. Vidal Dasilva, M. Fernändez-Perea, J. I. Larruquert Goicoechea, J. A. Mëndez Morales, J. A. Aznärez Candao, and J. M. Särchez Orejuela, "Narrowband filters for the extreme ultraviolet" U S Patent 12/429,944 (Aprilz4, 2009).

24. J. I. Larruquert, J. Opt. Soc. Am. A 18, 1406 (2001).

25. J. I. Larruquert, J. Opt. Soc. Am. A 18, 2617 (2001).

26. J. I. Larruquert and R. A. M. Keski-Kuha, Appl. Opt 38, 1231 (1999).

27. J. I. Larruquert and R. A. M. Keski-Kuha, Appl. Opt. 41, 5398 (2002).

28. J. I. Larruquert, J. Opt. Soc. Am. A, 19, 391 (2002).

29. J. I. Larruquert, Opt. Commum. 206, 259 (2002).

30. J. I. Larruquert, J. Opt. Soc. Am. A 21, 1750 (2004).

31. J. I. Larruquert, J. Opt. Soc. Am. A 19, 385 (2002).

32. J. I. Larruquert, J. Opt. Soc. Am. A 22, 1607 (2005).

33. J. I. Larruquert, J. Opt. Soc. Am. A 23, 1967 (2006). 